\title{
Multiresidue analysis of 184 pesticides in high-fat fish feed using a new generic extraction method coupled with gas and liquid chromatography-tandem mass spectrometry
}

\author{
Philipp Rudolf Michael Peter Eyring ${ }^{1 *} \mathbb{C}$, Susan Strange Herrmann ${ }^{2}$ and Mette Erecius Poulsen ${ }^{1}$
}

\begin{abstract}
Animal feed is typically plant-based and can contain pesticide residues. Methods for testing food and feed samples, such as the Quick Easy Cheap Effective Rugged Safe (QuEChERS) method or the Swedish Ethyl Acetate (SweEt) method, successfully extract many pesticide residues. However, nonpolar pesticides, such as organochlorine pesticides, show poor recovery when extracted from lipid-rich samples. The previously developed water-acetonitrile-heptane-solid-phase-extraction (WAHSPE) method shows better recoveries for the nonpolar pesticides but requires two injections per sample and per instrument. Here, we present a modified version of the WAHSPE method for pesticides in fish feed using one injection per sample and per instrument. Of the 184 pesticides tested, 179 met the European Union Legislation's validation criteria at a spike level of $50 \mu \mathrm{g} / \mathrm{kg}$, showing recoveries between 70 and 120\% and a relative standard deviation (RSD) below 20\%. Organochlorine pesticides accounted for 14 of the tested compounds.
\end{abstract}

Keywords: Multiresidue extraction method, Feed, Gas chromatography-tandem mass spectrometry, Lipid-rich sample, Liquid chromatography-tandem mass spectrometry, Organochlorine, Pesticide

\section{Introduction}

"Feed" is a general term describing any nourishment provided to animals, such as pigs, cows, and fish, and is mainly of plant origin [14]. Pesticides are used to ensure high yields and high quality crops. For food and environmental safety, the European Union (EU) established maximum residue limits (MRLs) for pesticide residues [13]. The MRLs established for food commodities also apply to crops intended for feed. Thus, all feed commodities also need to comply with the food laws.

In this study, it is crucial to monitor pesticide residues in feed samples. Common extraction procedures

\footnotetext{
*Correspondence: philipp.eyring@web.de

${ }^{1}$ National Food Institute, Technical University of Denmark, Kemitorvet,

2800 Kongens Lyngby, Denmark

Full list of author information is available at the end of the article
}

for pesticide analysis from feed samples include methods such as the Quick Easy Effective Rugged Safe (QuEChERS) method [1, 2, 5] and the Swedish ethyl acetate (SweEt) method [8]. Detection of compounds is typically performed by chromatography coupled to a mass spectrometer, such as gas chromatographytandem mass spectrometry (GC-MS/MS) and liquid chromatography-tandem mass spectrometry (LC-MS/ MS) $[1,2,5,10]$.

Although QuEChERS and SweEt are widely used methods and generally perform well, some analytes, such as organochlorine pesticides and other nonpolar pesticides, have low recoveries when extracted from lipid-rich samples [6]. We previously developed a method, "water-acetonitrile-heptane-solid-phase-extraction" (WAHSPE), that effectively addressed this problem [4]. However, for the analysis of pesticides in high-fat fish feed, the 
WAHSPE method requires multiple injections of each sample. Multiple injections are not optimal, because multiple injections mean lower overall sample throughput. Consequently, a modified version of the WAHSPE method was developed to reduce instrument time by requiring only one injection for each of the analytical techniques (GC-MS/MS or LC-MS/MS).

The modified method was validated for 184 pesticides, including 14 nonpolar organochlorine pesticides. The sample was a fish feed matrix with a high lipid content $(23 \%)$. In our previous work we validated the initial WAHSPE method using matrix-matched standard calibration. Analyte recoveries were typically in the range of $70-120 \%$ with relative standard deviations below $20 \%$. [4]. This article aims to further develop the WAHSPE method by minimizing the time from sample preparation to final result, while still obtaining good recovery and detection of highly polar and nonpolar pesticides in a lipid-rich matrix, thereby providing a more generic alternative to the QuEChERS and SweEt methods. This publication shows a streamlined workflow and its application for real fish feed samples.

\section{Materials and methods Chemicals}

Acetonitrile (HPLC grade S) was obtained from Rathburn (Walkerburn, UK). Heptane and formic acid were purchased from Merck (Darmstadt, Germany). Water was obtained from a Millipore Milli-Q water purification system (Molsheim, France). Ammonium formate was purchased from Sigma Aldrich (Buchs, Switzerland).

The analytical pesticide standards included in the study (purity $>96 \%$ ) were purchased from Dr. Ehrenstorfer (Augsburg, Germany) or Sigma Aldrich (Taufkirchen, Germany). The relevant pesticides are listed in the Additional file 1.

\section{Sample material}

The fish feed sample for the validation experiment, selected due to its high lipid content, was obtained from BioMar A/S (Brande, Denmark). The feed sample consisted of $2 \mathrm{~mm}$ pellets containing $22.8 \%$ crude fat and was called CPK 15 (brand name).

The 16 fish feed samples analyzed in this study were collected in 2017 by the National Reference Laboratories (NRL) within the EU. The samples originated from the following EU member states: Austria, Czech Republic, Denmark, Estonia, Germany, Greece, Hungary, Italy, Norway, Poland, Slovakia, and Spain.

\section{Materials}

Filter vials $(0.2 \mu \mathrm{m}$ pore size $)$ were obtained from Whatman, GE Healthcare (Princeton, USA). The
supelcleanTM SPE cartridge EZ-Pop NP was obtained from Supelco (St. Louis, USA). A Reax 2 shaker for end-over-end extraction was obtained from Heidolph (Schwabach, Germany). Primary secondary amine (PSA), $\mathrm{MgSO}_{4}$, and $\mathrm{C}_{18}$ sorbent were obtained from Agilent (Santa Clara, USA), Merck (Darmstadt, Germany), and International Sorbent Technology Ltd (Hengoed, UK), respectively. Single-use $15 \mathrm{~mL}$ and $30 \mathrm{~mL}$ polypropylene extraction tubes were obtained from Sarstedt (Nümbrecht, Germany). A Multifuge X3 FR centrifuge was obtained from Thermo Scientific (Waltham, USA). An OLE DICH Z 216 MK microcentrifuge was purchased from Hermle Labortechnik GmbH (Wehingen, Germany). Glass tubes $(16.0 \times 0.8-1.0 \mathrm{~mm})$ were obtained from VWR (Søborg, Denmark). A TurboVap LV Evaporator nitrogen degasser was obtained from Zymark (Hopkinton, USA). Micropipettes and $1.5 \mathrm{~mL}$ microcentrifuge tubes were obtained from Eppendorf (Hamburg, Germany).

\section{Sample preparation procedure}

Each sample (at least $100 \mathrm{~g}$ ) was cryo-milled (using liquid nitrogen) on a mill from Retsch (Haan, Germany) using a $1.0 \mathrm{~mm}$ mesh sieve. An aliquot of the milled sample $(5.0 \mathrm{~g})$ was placed in a $50 \mathrm{~mL}$ tube, and $10.0 \mathrm{~mL} 5 \%$ formic acid in acetonitrile, $10 \mathrm{~mL}$ Milli-Q water, and $10 \mathrm{~mL}$ heptane were added before the mixture was shaken end-over-end for $1 \mathrm{~h}$. A total of $5.0 \mathrm{~g}$ ammonium formate was added to induce phase separation, and the mixture was shaken manually for $1 \mathrm{~min}$, followed by centrifugation for $10 \mathrm{~min}$ at $5000 \times g$ and $20{ }^{\circ} \mathrm{C}$. Three phases (water, acetonitrile, and heptane) were formed, and the two organic phases were transferred to separate $15 \mathrm{~mL}$ tubes. A $1.0 \mathrm{~mL}$ aliquot of the acetonitrile phase was transferred to an Eppendorf vial containing $250 \mathrm{mg}$ PSA, $150 \mathrm{mg} \mathrm{MgSO}_{4}$, and $25 \mathrm{mg} \mathrm{C}_{18}$ for dispersive solid-phase extraction (dSPE) clean-up. The Eppendorf tube was shaken for $30 \mathrm{~s}$ and centrifuged at $10,000 \times g$ for $10 \mathrm{~min}$. Approximately $350 \mu \mathrm{L}$ of the supernatant was transferred to a filter vial $(0.2 \mu \mathrm{m}$ pore size). Then, $200 \mu \mathrm{L}$ of the filtered supernatant was transferred to a glass vial with a glass insert and acidified by adding $10 \mu \mathrm{L}$ of $1 \%$ formic acid in acetonitrile. A $1.0 \mathrm{~mL}$ aliquot of the heptane phase was transferred into a glass tube, and $1.0 \mathrm{~mL}$ of pure acetonitrile was added. A gentle stream of nitrogen $\left(40{ }^{\circ} \mathrm{C}\right)$ was used to evaporate the heptane phase until only the acetonitrile and residual lipids remained. The acetonitrile and the residual lipids were cleaned-up using an EZ-Pop NP SPE column as follows: the column was pre-conditioned with $10 \mathrm{~mL}$ acetone and dried for $10 \mathrm{~min}$ by vacuum. The acetonitrile and the residual lipids were loaded onto 
the column. The retained analytes were eluted into a $20 \mathrm{~mL}$ glass test tube using $15 \mathrm{~mL}$ acetonitrile. The eluate was evaporated using a nitrogen stream at $40{ }^{\circ} \mathrm{C}$ for approximately $2 \mathrm{~h}$, until a sample of approximately $1 \mathrm{~mL}$ was obtained.

A $100 \mu \mathrm{L}$ aliquot of the acetonitrile eluate (cleaned heptane phase) and $100 \mu \mathrm{L}$ of the filtered and acidified acetonitrile extract were mixed in an Eppendorf vial. Then, $100 \mu \mathrm{L}$ of this mixture was mixed with $100 \mu \mathrm{L}$ TPP (triphenyl phosphate) solution $(0.2 \mu \mathrm{g} / \mathrm{mL}$ TPP in acetonitrile) in an autosampler vial and analyzed by GC-MS/MS and LC-MS/MS.

\section{Method validation \\ Spiking procedure}

Cryo-milled and sieved fish feed samples (5.0 g) were spiked with a stock solution containing 184 pesticides at three different levels $(5,10$, and $50 \mu \mathrm{g} / \mathrm{kg})$, with six replicates for each level.

\section{Recoveries}

Quantitation was based on the quantifier ion peak area divided by the peak area of the internal standard (TPP) and compared to a procedural calibration curve, which was described by a best-fit line. Seven procedural calibration points for each pesticide were included, ranging from 5.0 to $267 \mu \mathrm{g} / \mathrm{kg}$. The procedural standards were prepared by spiking, with calibration solutions, a milled and sieved blank fish feed sample and following the sample preparation procedure (see Section "Sample preparation procedure" after milling and sieving).

Unfortunately, the unspiked blank sample contained low amounts of the pesticides fenpropidin, permethrin, pirimiphos-methyl, and propargite. Thus, the blank sample peak areas were subtracted from all spiked sample peaks for these pesticides. The content in the blank sample was also subtracted from the content in the calibration samples before preparation of the calibration curves.

\section{LC-MS/MS analysis}

For LC-MS/MS analysis, a Sciex Q-TRAP 6500 MSMS system linked to a Waters Acquity LC-system (degasser, pump, autosampler, and column compartment) was used. The column oven temperature was $40{ }^{\circ} \mathrm{C}$, and the injection volume was $10 \mu \mathrm{L}$. For separation, a Sielc Obelisc R HPLC column (5 $\mu \mathrm{m}$ particle size, $2.1 \times 150 \mathrm{~mm}$ ) was used. The mobile phase consisted of Eluent A (water with $0.4 \%$ formic acid and $20 \mathrm{mM}$ ammonium formate) and Eluent B (acetonitrile). The mobile phase gradient was as follows: linear gradient from 20 to $80 \% \mathrm{~B}(0-4 \mathrm{~min})$, isocratic at $80 \% \mathrm{~B}$ (4-12 min), linear gradient to $20 \%$ B (12-12.5 min), and isocratic $20 \% \mathrm{~B}$ for $7.5 \mathrm{~min}(12.5-20 \mathrm{~min})$ to prepare the column for the next injection. Electrospray ionization (ESI) source parameters were as follows: curtain gas, $40 \mathrm{~L} / \mathrm{h}$; collision gas, medium; ion spray voltage, $3000 \mathrm{~V}$; source temperature, $500{ }^{\circ} \mathrm{C}$; ion source gas $1,40 \mathrm{~L} / \mathrm{h}$; ion source gas 2, $60 \mathrm{~L} / \mathrm{h}$. The dwell time was $10 \mathrm{~ms}$, and the entrance potential (EP) was $10 \mathrm{~V}$. The mass spectrometer was operating in multiple reaction monitoring (MRM) mode. To identify the pesticides, retention time and at least two product ions were acquired. MRM transitions are listed in Additional file 2: Table S1. One product ion was used for quantitation (quantifier ion), and the other product ion was used for identification (qualifier ion). Quantitation was based on the quantifier ion peak area divided by the peak area of the internal standard (TPP) and compared to the procedural calibration curve.

\section{GC-MS/MS analysis}

GC-MS/MS analysis was performed on a TSQ 8000 Evo Triple Quadrupole Mass Spectrometer paired with a TRACE 1310 GC and a Thermo Scientific TriPlus RSH autosampler. For chromatographic separation, a DB5-MS column was employed. The instrument was operating in MRM mode. Electron energy was $70 \mathrm{eV}$, the source temperature was $180^{\circ} \mathrm{C}$, and the transfer line temperature was $250{ }^{\circ} \mathrm{C}$. The injection volume was $5 \mu \mathrm{L}$. For each pesticide, two sets of precursor and product ions were determined. One ion was for quantification and at least one ion was for qualification. MRM transitions are listed in Additional file 2: Table S1. The temperature program was as follows: $60^{\circ} \mathrm{C}$ steady for $0.8 \mathrm{~min}$, a gradient of $30^{\circ} \mathrm{C} /$ min until $180{ }^{\circ} \mathrm{C}$, a gradient of $5{ }^{\circ} \mathrm{C} /$ min until $280{ }^{\circ} \mathrm{C}$, a gradient of $40{ }^{\circ} \mathrm{C} / \mathrm{min}$ until $300^{\circ} \mathrm{C}$, a gradient of $120^{\circ} \mathrm{C} /$ min until $310^{\circ} \mathrm{C}$. Quantitation was based on the quantifier ion peak area divided by the peak area of the internal standard (TPP) and compared to the procedural calibration curve.

\section{Availability of data and materials}

The datasets used and analyzed during the current study are available from the corresponding author upon reasonable request.

\section{Results and discussion \\ Method development}

The method that was developed was based on the method reported in Eyring et al., but improvements were made by decreasing preparation and instrument time [4]. The decrease in instrument time was achieved by decreasing the number of injections for each extract from 


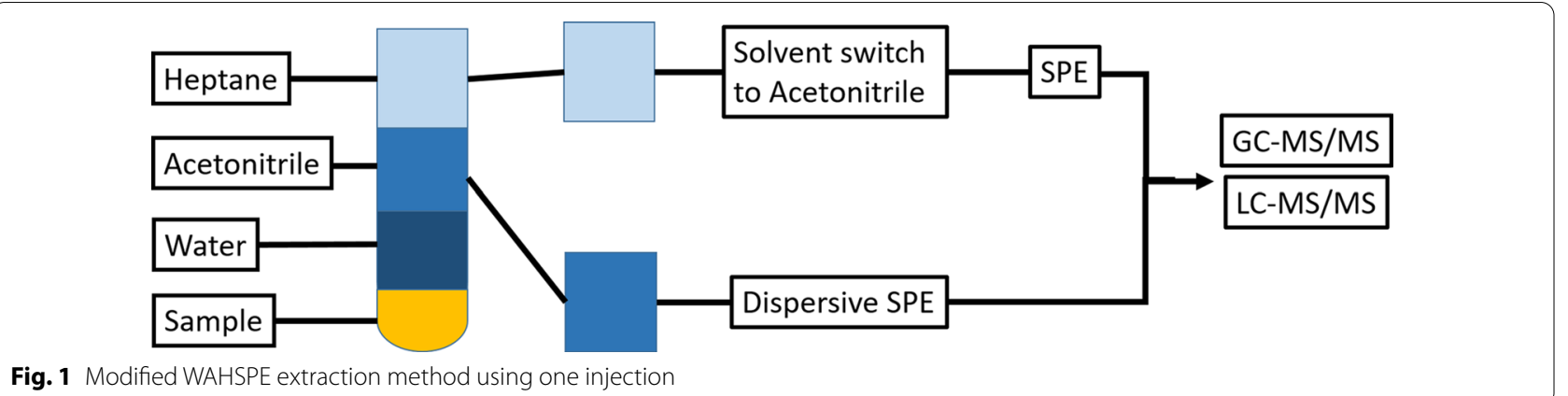

Fig. 1 Modified WAHSPE extraction method using one injection

two to one. The sample was extracted with three solvents (water, acetonitrile, and heptane) in the new method. A solvent shift to acetonitrile was done before the clean-up of the heptane phase. After the clean-up, the two acetonitrile extracts for each sample were mixed and analyzed (see Fig. 1).

\section{Method validation}

A validation study for 184 pesticides in fish feed with $23 \%$ lipid content was carried out to determine the performance of the modified WAHSPE method. The validation study involved extraction and analysis of the pesticides from fish feed samples spiked at three levels $(5,10$, and $50 \mu \mathrm{g} / \mathrm{kg})$ with six replicates at each level.

The validation parameters for the developed method were linearity, selectivity, limit of quantitation (LOQ), repeatability, accuracy, and precision. Matrix effects were taken into account using a calibration curve of procedural standards.

Linearity was tested by injecting seven different concentrations of each pesticide, plotting calibration curves, and calculating the correlation coefficients $\left(R^{2}\right)$ for a linear fit. Selectivity was assessed by detecting the quantifier ion and at least one qualifier ion for each pesticide. The LOQ was determined by injecting six replicates of blank samples that were spiked at concentrations of 5,10 , and $50 \mu \mathrm{g} / \mathrm{kg}$. The lowest of these concentrations that met the acceptance criteria (recovery of $70-120 \%$ and relative standard deviation (RSD) below 20\%) was defined as the LOQ. Recoveries below $70 \%$ were accepted if the RSD was below $20 \%$, but the concentrations found in these cases were corrected for the low recovery as described in SANTE/12682/2019 [3].

The repeatability was tested by performing six replicates within one day (intra-day variability) for each

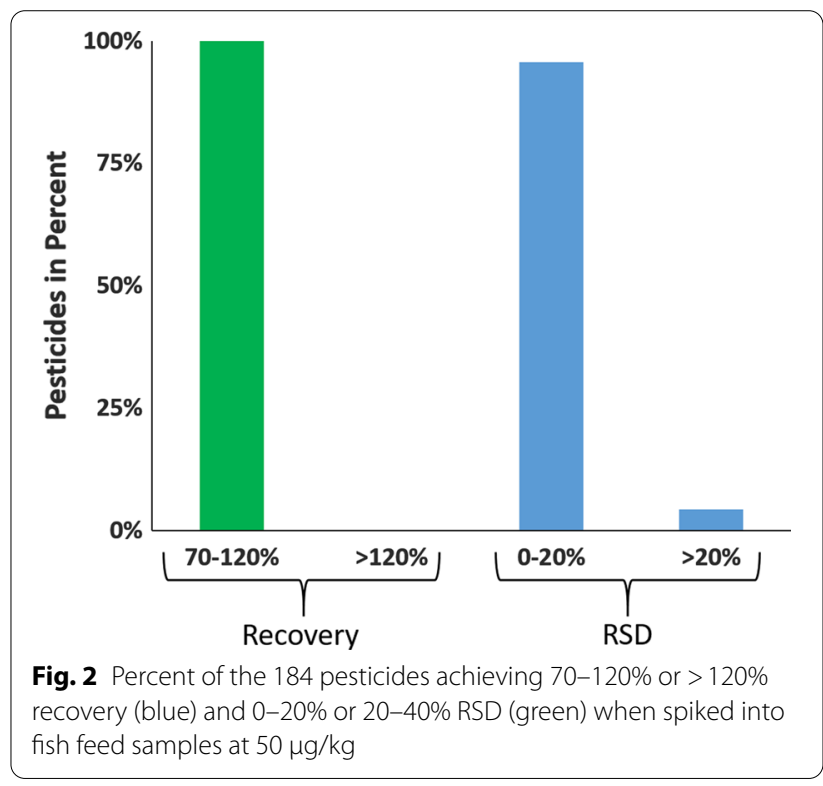

concentration. Repeatability was evaluated by measuring the RSDs of recovery replicates studied for each pesticide. The accuracy was determined from the recoveries from the spiked blank samples. The precision of the method for each pesticide was evaluated by determining the RSDs on the recoveries for the replicates.

All analytes showed good linearity, since all calculated $\mathrm{R}^{2}$ values were $\geq 0.98$. The LOQs for the 184 pesticides were between 5 and $50 \mu \mathrm{g} / \mathrm{kg}$ (see Additional file 3: Table S2). An LOQ of $50 \mu \mathrm{g} / \mathrm{kg}$ was achieved for 179 pesticides (see Fig. 2). There were no pesticides with recoveries above $120 \%$, but some recoveries were not possible to validate due to RSD values higher than 
Table 1 Recoveries and repeatability values (RSDs in parentheses) for the analysis of organochlorine pesticides at a $50 \mu \mathrm{g} / \mathrm{kg}$ spike level

\begin{tabular}{ll}
\hline Compound & $\begin{array}{l}\text { Percent total } \\
\text { recovery (RSD) }\end{array}$ \\
\hline Dieldrin & $93(29)$ \\
Chlordane alpha-cis & $87(15)$ \\
Chlordane gamma-trans & $94(12)$ \\
DDD pp & $91(13)$ \\
DDE pp & $94(16)$ \\
DDT op & $97(20)$ \\
DDT pp & $97(17)$ \\
Endosulfan sulfate & $98(16)$ \\
Endosulfan alpha & $91(11)$ \\
Endrin & $88(16)$ \\
HCH, alpha & $88(11)$ \\
HCH, beta & $88(17)$ \\
Heptachlor & $93(19)$ \\
Heptachlor epoxide & $92(15)$ \\
\hline
\end{tabular}

$20 \%$. These pesticides were dichlorvos, dieldrin, hexaconazole, profenofos, and propargite. A possible reason for these high RSDs may be interfering compounds, which co-elute with the analytes $[9,11,12]$.

When analyzing the combined heptane and acetonitrile phases, more interfering matrix compounds may have been present than when the heptane and acetonitrile phases were analyzed separately. Different cleanup sorbents could be applied to reduce the amount of interfering matrix compounds, though the benefit compared to the higher effort should always be considered. The interfering matrix level was found to be acceptable overall for the clean-up method presented here. Thirteen of the 14 organochlorine pesticides were successfully validated at the spike level of $50 \mu \mathrm{g} / \mathrm{kg}$ (see Table 1). The QuEChERS method typically results in low recoveries for organochlorine pesticides from lipid-rich samples [6]. Generally, the SweEt method performs well when used to analyze pesticides in fruits and vegetables that have a low lipid content [8]. The SweEt method was tested, in a modified version, on samples with a high lipid content. Cyclohexane was used as the extraction solvent and gel permeation chromatography was added as a clean-up step [7]. Unfortunately, this additional step made the method very time-consuming.

The WAHSPE method used in the present study in general showed good performance for the analysis of nonpolar analytes and pesticides in lipid-rich matrices. Therefore, the modified WAHSPE method excels exactly where QuEChERS and SweEt fall short. Furthermore, the improvement of the WAHSPE procedure from our previous work [4] allows for increased sample throughput.

\section{Application of the method to real samples and achieved benefits}

The validated method was applied to the analysis of 16 real-world fish feed samples. This analysis was intended to demonstrate the applicability of the method to realworld samples and the potential for implementation of the method in routine monitoring of pesticides. Of the 16 samples, eight contained pesticides above the respective LOQs (see Table 2). Bromuconazole was found in four samples, difenoconazole and fludioxonil were each found in two samples, and 2-phenyl-phenol, cypermethrin, deltamethrin, diphenylamine, and fenazaquin were each found in one sample. Even though fish feed represents a complex sample for which it is difficult to obtain acceptable pesticide multi-residue analysis results, especially for nonpolar pesticides such as organochlorines, the present study showed that a modified WAHSPE method, combined with GC-MS/MS and LC-MS/MS analysis, is an excellent tool for analyzing pesticides of a wide polarity range in lipid-rich matrices. Compared to other extraction methods, such as QuEChERS and SweEt, the proposed method demonstrates improved recoveries of nonpolar organochlorine pesticides and provides acceptable recoveries for other pesticides at the same time. Furthermore, this modified version of the WAHSPE method requires fewer injections than the initial version, thus matching the SweEt and QuEChERS methods regarding efficiency. Acceptable values for precision, LOQs, and accuracy were obtained, showing that the method is suitable for regulatory and routine pesticide multi-residue analysis in lipid-rich matrices. 


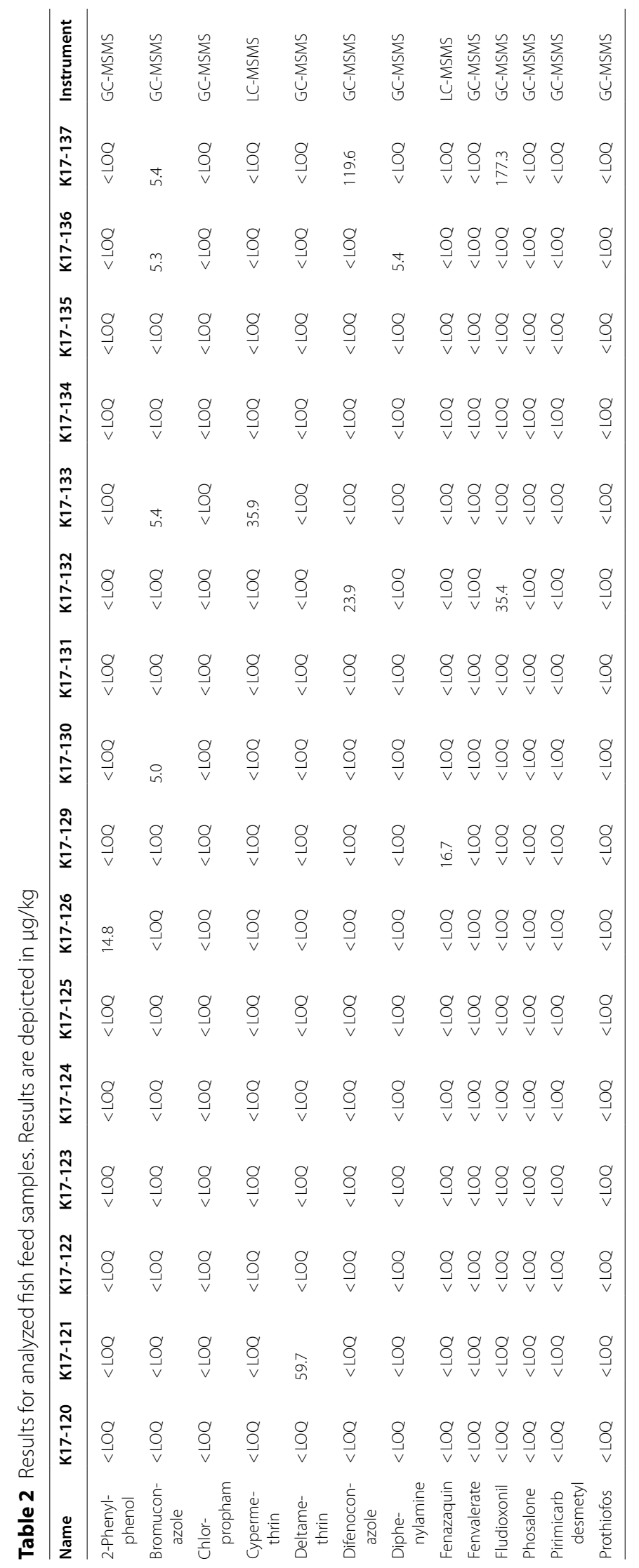




\section{Supplementary Information}

The online version contains supplementary material available at https://doi. org/10.1186/s13765-021-00610-9.

Additional file 1: Pesticide standards used in this work.

Additional file 2: Table S1. MRM and retention times of 184 pesticides.

Additional file 3: Table S2. Validation results and LOQs.

\section{Acknowledgements}

We thank Helen Fodnæss for proof reading. This work was supported by the Ministry of Environment and Food of Denmark [Fødevareforlig 3, 2015-2018]. The funding source has no role in the study design, in the collection, analysis, and interpretation of data, in the writing of the report, nor in the decision to submit the article for publication.

\section{Authors' contributions}

PE: planning experiments, executing experiments, data analysis, data evaluation; SSH: planning experiments, support and advice in LC-MS/MS data analysis, support in result evaluation; MEP: planning experiments, support and advice in GC-MS/MS data analysis, support in result evaluation. All authors read and approved the final manuscript.

\section{Availability of data and materials}

The datasets used and/or analysed during the current study are available from the corresponding author on reasonable request.

\section{Declarations}

\section{Competing interests}

The authors of the paper do not declare a competitive fiscal gain.

\section{Author details}

${ }^{1}$ National Food Institute, Technical University of Denmark, Kemitorvet, 2800 Kongens Lyngby, Denmark. ${ }^{2}$ Rigshospitalet, Valdemar Hansens Vej, 2600 Glostrup, Denmark

Received: 14 November 2020 Accepted: 19 April 2021

Published online: 26 April 2021

\section{References}

1. Anastassiades M, Lehotay SJ, Štajnbaher D, Schenck FJ (2003) Fast and easy multiresidue method employing acetonitrile extraction/partitioning and. J AOAC Int 86:412-431. https://doi.org/10.2478/s11687-011-0011-9
2. Anastassiades M, Scherbaum E, Taşdelen B, Štajnbaher D (2007) Recent developments in QuEChERS methodology for pesticide multiresidue analysis. In: Ohkawa H, Miyagawa H, Lee PW (eds) Pesticide chemistry: crop protection, public health environmental safety. Wiley, Weinheim

3. European Commission (2019) Method validation procedures for pesticide residues analysis in food and feed. Sante/12682/2019

4. Eyring P, Tienstra M, Mol H et al (2021) Development of a new generic extraction method for the analysis of pesticides, mycotoxins, and polycyclic aromatic hydrocarbons in representative animal feed and food samples. Food Chem. https://doi.org/10.1016/j.foodchem.2021.129653

5. Lehotay SJ, Maštovská K, Lightfield AR (2005) Use of buffering and other means to improve results of problematic pesticides in a fast and easy method for residue analysis of fruits and vegetables. J AOAC Int 88:615-629

6. Lehotay SJ, Maštovská K, Yun SJ (2005) Evaluation of two fast and easy methods for pesticide residue analysis in fatty food matrixes. J AOAC Int 88:630-638. https://doi.org/10.1093/jaoac/88.2.630

7. NMKL (2013) Pesticide residues. Analysis in foods with ethyl acetate extraction using gas and liquid chromatography with tandem mass spectrometric determination

8. Pihlström T, Blomkvist G, Friman P et al (2007) Analysis of pesticide residues in fruit and vegetables with ethyl acetate extraction using gas and liquid chromatography with tandem mass spectrometric detection. Anal BioanalChem 389:1773-1789. https://doi.org/10.1007/s00216-007-1425-6

9. Rahman MM, Abd El-Aty AM, Shim JH (2013) Matrix enhancement effect: a blessing or a curse for gas chromatography?-a review. Anal ChimActa 801:14-21. https://doi.org/10.1016/j.aca.2013.09.005

10. Shin Y, Lee J, Kim JH (2018) A simultaneous multiresidue analysis for 203 pesticides in soybean using florisil solid-phase extraction and gas chromatography-tandem mass spectrometry. ApplBiolChem 61:543-548. https://doi.org/10.1007/s13765-018-0388-y

11. Sugitate K, Nakamura S, Orikata N et al (2012) Search of components causing matrix effects on GC/MS for pesticide analysis in food. J PesticSci 37:156-163. https://doi.org/10.1584/jpestics.D11-048

12. Sugitate K, Saka M (2015) Decrease in the matrix enhancement effect on pesticides analysis with GC-MS using new types of solid-phase extraction column. J PesticSci 40:87-91. https://doi.org/10.1584/jpestics.D15-010

13. The European Comission (2017) Regulation (EU) $2017 / 625$ of the European parliament and of the council

14. Tienstra M, Mol HGJ (2018) Application of gas chromatography coupled to quadrupole-orbitrap mass spectrometry for pesticide residue analysis in cereals and feed ingredients. J AOAC Int 101:342-351. https://doi.org/ 10.5740/jaoacint.17-0408

\section{Publisher's Note}

Springer Nature remains neutral with regard to jurisdictional claims in published maps and institutional affiliations.

\section{Submit your manuscript to a SpringerOpen ${ }^{\circ}$ journal and benefit from:}

- Convenient online submission

$\checkmark$ Rigorous peer review

- Open access: articles freely available online

- High visibility within the field

- Retaining the copyright to your article

Submit your next manuscript at springeropen.com 